\title{
On the Trade-off between Spectrum Efficiency and Transmission Cost in Traditional and SFN-based Broadcast Television
}

\author{
Rolando E. Bettancourt \\ Department of Engineering and Public Policy \\ Carnegie Mellon University \\ Pittsburgh, PA 15213 \\ Email: rbettancourt@cmu.edu
}

\author{
Jon M. Peha \\ Department of Engineering and Public Policy \\ Department of Electrical and Computer Engineering \\ Carnegie Mellon University \\ Pittsburgh, PA 15213 \\ Email: peha@cmu.edu
}

\begin{abstract}
This paper evaluates two alternatives to today's noise-limited single-transmitter broadcast television systems, either of which would improve spectrum efficiency. One alternative is to increase the transmit power of each broadcaster's only transmitter. The other alternative is to replace that single transmitter with a multi-transmitter single frequency network (SFN). For both approaches, this paper calculates the relationship between the maximum achievable spectrum efficiency and the cost required to achieve that efficiency as a function of the most important design parameters. Results suggest that increasing power of traditional single-transmitter broadcasters could reduce the amount of spectrum needed for TV by roughly $30 \%$, and would be cost-effective today through much of the U.S. A switch to SFNs could reduce the amount of spectrum needed for TV by roughly $60 \%$, but at a higher cost. Results suggest that the SFN approach could be cost-effective in the most densely populated regions where spectrum is most valuable.
\end{abstract}

Index Terms-Spectrum, Single Frequency Networks, Television, DTV, SFN, LPLT.

\section{INTRODUCTION}

Spectrum policies have long minimized the cost of television transmission per area covered while ignoring the opportunity cost of the spectrum. They do that in two ways. They do that in part by choosing the single-transmitter broadcasting approach, in which elevated sites have to transmit in the range of tenths to hundreds of $\mathrm{kW}$, and they do that in part by making sure distances between coverage areas are so large that the effect of interference is negligible.

One alternative to this is the use of low-power low-tower (LPLT) Single Frequency Networks (SFN), where multiple synchronous transmitters send the same signal over the same frequency channel, both at much lower heights and transmit power [1-3]. With SFNs, the building and operational costs are greater, but so is the potential spectrum efficiency. Also, either with SFNs or traditional broadcasting we could reduce the distance between coverage areas and tolerate the additional interference by increasing signal strength at the edge of coverage. However, increasing spectrum efficiency by tolerating additional interference would also increase the cost to broadcasters. Setting the distance between broadcasters such that interference is negligible might be the right strategy if the goal is to minimize broadcast stations' transmission costs, or if spectrum is considered to be so plentiful that its cost can be ignored. That may have been the world we lived in when regulatory bodies started granting TV licenses, but it is certainly not the world today [4], which is in part why our work revisits this issue and examines the cost effectiveness of an interference-limited approach.

Lately, the use of SFNs has been actively discussed. In the U.S., a distributed transmission architecture switchover was proposed to enable a more efficient use of the television spectrum in the context of the 2010 U.S. National Broadband Plan [5,6]. More recently, the use of SFNs has been required in the discussions of the candidate technologies for an upcoming new Advance Television System Committee (ATSC) 3.0 TV transmission standard [7, 8]. In Europe, the use of LPLT SFNs has being considered as a possible way to reclaim a significant amount of spectrum for broadband use in the discussion of the future of the UHF television band $[3,9,10]$.

In this paper, we examine the trade-offs between efficiency of spectrum use and the broadcast transmission cost required to achieve that efficiency in a suitable spectrum band that is only used for television. We do this with an analytical model that assumes a single television channel that is used by multiple identical broadcasters, each of which serves a fixed amount of area, and these areas are served through either a traditional single-transmitter approach or by a SFN. As this band is used only for television, we measure the efficiency of spectrum use as the fraction of total area that falls within a TV coverage area. In this model we assume Orthogonal Frequency Division Multiplexing (OFDM) technology and its typical parameters for fixed reception, but it is not tied to a specific broadcast television standard.

In terms of costs, we assume that a traditional television station is in place, and estimate the net present value (NPV) of both the cost of upgrading infrastructure, if any, and the change in long-term operating costs when using a cost-minimizing design for each broadcaster. With traditional TV, any change in cost comes from an increase in transmit power, and nearly 
all of this is the cost of increased energy consumption. For SFNs, a new infrastructure must be deployed and the design choice that most affects cost is the number of transmitters per area covered that each broadcaster operates.

The paper is organized as follows. Section II describes the most relevant literature related to spectrum efficiency with the use of SFNs. Section III develops a theoretical analytical model by obtaining the separation distance between two broadcasters' coverage areas. Section IV presents the main results of this paper and potential policy implications, while Section V focuses on concluding remarks.

\section{RELATED WORK}

Previous studies have proposed a more efficient use of the television spectrum by changing the transmission architecture [5,11-13]. In 2009, the Cellular Telephone Industries Association (CTIA) and the Consumer Electronics Association (CEA) proposed to the US Federal Communications Commission (FCC) an ATSC 8VSB based switchover to SFNs [5]. The proposal suggested that with SFNs the spectral separation distances between TV spectrum licensees can be reduced, and this could free up spectrum by repacking them into a smaller portion of the existing television spectrum without significantly changing the coverage areas or capabilities of current TV broadcasters. However, neither cost nor benefits of such a switchover were quantified. In this work, we aim to quantify these co-channel separation distances when using SFNs, and the impact on spectrum efficiency and cost. Moreover, the ATSC 8VSB modulation may not be the best suited for SFN operation anyway [14-16] so this paper considers the use of OFDM-based transmission [8, 16-19].

Other studies have also considered the use of SFNs, but with industry structures that are different from what we have today in many parts of the world [11-13]. For example, today in the U.S. each broadcaster operates within its own spectrum block, and serves whatever area can be reached by its transmitter. Instead, all broadcast stations in a given region could operate through a single SFN provider with access to infrastructure and a block of spectrum much larger than $6 \mathrm{MHz}$, as considered in [11]. In [11] authors focus only on spectrum efficiency, and they conclude that today's U.S. television needs could be met with just $85 \mathrm{MHz}$ when using a LTE eMBMS SFN, as opposed to the $300 \mathrm{MHz}$ currently allocated in the US. In their results, much of the improvement comes from either trunking efficiencies of aggregating multiple variable-bit-rate TV streams, or from considering that total area between coverage areas is negligible compared to total area covered. In contrast, we consider the case where technology changes, but without a fundamental change in what a broadcaster does or in the industry structure. Specifically, we assume that each broadcast station will continue to operate its own frequency channel without significant change to its coverage area, and that most TV viewers will be served multiple competing broadcasters. In addition, rather than simply looking at potential gains in spectrum efficiency without consideration of cost, we analyze this trade-off.
In a similar way, the authors in $[12,13]$ build upon the work in [11] and look into the difference in spectrum consumption between typical urban and rural environments for an eMBMS-based SFN operation that comes from upgrading existing cellular operators' transmitter sites. They conclude that significant spectrum savings can be obtained in urban areas, where transmitter sites are plentiful, but they find only limited savings can be obtained in rural areas due to large ISDs between existing rural transmitters, which hurts eMBMS's achievable spectral efficiency (bps/Hz). In contrast, we allow infrastructure to vary freely with spectrum efficiency, and, as opposed to eMBMS case, we consider values for OFDM parameters that maximize spectral efficiency over fixed reception and large ISDs [20].

On the other hand, Europe is also considering the possible use of SFNs. Studies led by the European Commission and the Electronic Communications Committee (ECC) of the European Conference of Postal and Telecommunications Administrations (CEPT) have recently discussed the future of the UHF band and the possibility of a progressive re-farming $[3,9,10]$. While defining the next generation terrestrial broadcasting, the ECC analyzes the cost and benefits of moving to what they call a converged platform that would permit mobile and terrestrial TV broadcast services to share a common infrastructure with much higher spectrum efficiency [3]. Although they expect that substantial spectrum could be freed through a SFN transition, the cost-benefit analysis in [3] concludes that the incremental benefits and costs are still uncertain: a new review is recommended within the next 3 to 5 years, alongside with further technical analysis on the feasibility and cost of cochannel SFNs with separation distances close to $0 \mathrm{~km}$. In this context, several stakeholders' reports were produced [21-26], however, there is no widely accepted estimates of the costs or the potential gains in spectrum efficiency so our paper aims to address some of these open issues.

\section{Model Development}

In this section, we calculate the maximum spectrum efficiency that can be obtained when identical broadcast networks are deployed in a frequency channel, for both traditional broadcasters and SFNs. In general, spectrum efficiency is a function of both the size of the broadcasters' coverage areas and the separation distance between those areas. We define spectrum efficiency as the maximum achievable fraction of area covered over a region that is much larger than the coverage area of a single broadcaster.

For a meaningful comparison, we must define coverage area such that if a SFN broadcaster and traditional broadcaster have the same size coverage area, it is reasonable to view them as being equally effective at bringing television to their viewers. One challenge is that the spatial distribution of the received signal strength across coverage areas of SFNs and traditional broadcasters are very different, and we must define coverage in a way that if a SFN broadcaster and traditional broadcaster have the same size coverage area, it is reasonable to view 
them as being equally effective at bringing television to their viewers.

We define the required coverage area in terms of coverage probability, which is a function of the Signal-to-Interferenceplus-Noise Ratio (SINR) for a receiver at a particular location. In SFNs, the total received signal is the power-sum of multiple OFDM useful and interfering components. Hence, we calculate coverage probability through an approximation of the SINR distribution in a general setting that considers multiple possibly correlated useful and interfering signals.

In this section, we develop analytical expressions for separation distances between broadcasters' coverage areas for a given network design of each type, such that spectrum efficiency can be calculated. For traditional broadcasters, we obtain minimum separation distance, for a given transmit power and required coverage area. For SFNs, we do this as a function of ISD.

\section{A. Definition of Coverage}

For traditional broadcasting, we would typically say that any point is within the coverage area if coverage probability $q$ for the broadcaster's signal exceeds some fixed threshold $q_{\text {thr }}$. This means that coverage probability will be close to $100 \%$ near the transmitter, and will gradually decrease with distance from the transmitter until the threshold is reached at the edge of coverage. However, if we adopt the same definition with an SFN, and then design the least expensive SFN that can provide a coverage probability greater than $q_{\mathrm{thr}}$ in all points that are covered by the traditional broadcaster, the resulting SFN's coverage would be far worse than that for the traditional broadcaster. In this SFN, a large portion of the coverage area would have a coverage probability close to $q_{\mathrm{thr}}$, including points near the center; whereas for the traditional broadcaster, this would only occur near the edge. Thus, to make our definition of coverage more appropriate for both SFNs and traditional broadcasters, we use two different coverage probability thresholds: a lower threshold $q_{\text {thr }}$ near the edge of coverage, and a higher threshold $q_{\text {thr }}^{\prime}$ further inside. Any point with coverage probability greater than the higher threshold $q_{\mathrm{thr}}^{\prime}$ is considered covered. If the set of points with coverage probability greater than $q_{\mathrm{thr}}^{\prime}$ form a contiguous area, as we would expect in a SFN that is designed to have a large contiguous high-quality coverage area, then the contiguous set of points surrounding this area with $q_{\mathrm{thr}}<q<q_{\mathrm{thr}}^{\prime}$ are also considered to be within the coverage area.

For traditional broadcasters, the reference antenna currently used for coverage calculations (e.g. by ITU, FCC, others) is a directional antenna with a predefined radiation pattern [27, 28]. In this work, we maintain this antenna definition for the case of traditional broadcasting, but for the case of SFNs, our reference antenna is omni-directional. An important feature of our scenario is that we consider that every broadcaster has its own frequency channel and multiple transmitters, and the location of each broadcaster's transmitters may have different throughout the coverage area, i.e. they might not be co-located. Hence, it may be impractical for many viewers to point an antenna in a direction that is near optimal for all channels.
With omni-directional antennas, viewers greatly benefit from signal diversity, while avoiding any need of precisely pointing and/or reorienting antennas based on the location and/or the frequency of transmitters of different TV stations.

\section{B. Coverage Probability Calculation}

Considering a spectrum band only used for television, the coverage probability $q$ at any given point can be expressed as

$$
\begin{aligned}
q \stackrel{\text { def }}{=} & \operatorname{Pr}\left\{\gamma \geq \gamma_{\text {min }}\right\} \\
=\operatorname{Pr} & \left\{\sum_{j=1}^{J} w\left(\tau_{j}\right) S_{j} \geq \gamma_{\min } N_{0}+\gamma_{\min } \sum_{k=1}^{K} U_{k}\right. \\
& \left.+\gamma_{\text {min }} \sum_{j=1}^{J}\left[1-w\left(\tau_{j}\right)\right] S_{j}\right\} \\
=\operatorname{Pr}\left\{S \geq \gamma_{\min }\left(N_{0}+U\right)\right\} &
\end{aligned}
$$

where $\operatorname{Pr}\{A\}$ is the probability of event $\mathrm{A}, S_{j}$ and $U_{k}$ represent the received power from the $j$ th wanted transmitter and the $k$ th interferer respectively, and therefore $S=\sum w\left(\tau_{j}\right) S_{j}$ and $U=\sum U_{k}+\sum\left[1-w\left(\tau_{j}\right)\right] S_{j}$ are the total received wanted TV signal and the total unwanted signal (co- or adjacentchannel) respectively, and $N_{0}$ is the noise input power of the receiver, all of which are in the linear domain. The function $w\left(\tau_{j}\right)$ is the equalizer weighting function of the OFDM receiver, and represents the constructive portion of the $j$ th signal with relative delay $\tau_{j}$. For traditional broadcasting, $J=1$ and $w\left(\tau_{1}\right)=1$. For SFNs, we assume that $w\left(\tau_{j}\right)=1$, which is reasonable as long as there exists a guard interval (GI) duration $T_{g}$ such that $w\left(\tau_{j}\right) \approx 1$ across the entire coverage area [20]. This occurs when the fraction GI over the total OFDM symbol length is small, and ISD is much smaller compared to the distance that a signal can travel during one GI [29]. For example, for $T_{g}=260 \mu \mathrm{s}$ which is equivalent to approximately a $1 / 16$ fraction of a $32 \mathrm{~K}$ FFT symbol period in a $6 \mathrm{MHz}$ channel [20], would meet these requirements for a bandwidth $B \leq 8 \mathrm{MHz}$ and a ISD $\ll 80 \mathrm{~km}$. This includes the range of parameters considered in this work.

To evaluate $q=\operatorname{Pr}\left\{\gamma>\gamma_{\min }\right\}$, the probability distribution of the SINR $\gamma$ needs to be derived. For this, we assume that $S_{j}$ and $U_{k}$ are modeled as possibly correlated signals coming from multiple transmitters simultaneously, but with different path losses and possibly different antenna gains. Specifically, $S_{j}$ and $U_{k}$ are part of a set of $L=J+K$ correlated lognormal random variables (RV) $\left\{\Omega_{i}\right\}_{i=1}^{L}$ with parameters $\mu_{\Omega_{i}}$ and standard deviation $\sigma_{\Omega_{i}}$ (both in $\mathrm{dB}$ scale), i.e. each has a probability density function (PDF)

$$
f_{\Omega_{i}}(\omega)=\frac{\xi}{\omega \sqrt{2 \pi} \sigma_{\Omega_{i}}} \exp \left[-\frac{\left(\xi \log (\omega)-\mu_{\Omega_{i}}\right)^{2}}{2 \sigma_{\Omega_{i}}^{2}}\right],
$$

where $\mu_{\Omega_{i}}=P_{i}+g\left(\alpha_{i}\right)-L\left(d_{i} ; h_{i}\right)[\mathrm{dB}]$ is the median received power from the ith transmitter, $P_{i}$ and $h_{i}$ are transmit power and transmit antenna height respectively, the function $L\left(d_{i} ; h_{i}\right)$ represents the median path loss which is a function of the distance $d_{i}$ between transmitter and receiver, and $g\left(\alpha_{i}\right)$ 
is the gain of the receive antenna. Finally, the parameter $\xi=$ $10 / \ln (10)$ acts as a normalization factor. We assume a constant cross-correlation model [30,31]; thus, the cross-correlations in the decibel scale between all wanted-to-wanted, interfering-tointerfering and wanted-to-interfering components are identical with correlation coefficient $\rho[30,32]$ and all signals share the same standard deviation $\sigma_{\Omega_{i}}=\sigma$ [20].

To obtain the probability distribution of $S$ and $U$, we make the common assumption that the sum of log-normal RVs is well approximated by another log-normal RV, as exact closed-form expressions for the log-normal sum distribution do not exist to date [33]. Then, we follow the SchwartzYeh (SY) method [34] to recursively obtain, in pairs, the parameters $\left\{\mu_{\mathrm{S}}, \sigma_{\mathrm{S}}\right\}$ and $\left\{\mu_{\mathrm{U}}, \sigma_{\mathrm{U}}\right\}$ of the resulting log-normal sum distributions of $S$ and $U$. Therefore given two lognormal RVs $\Omega_{1}$ and $\Omega_{2}$ with correlation coefficient $\rho_{\Omega_{1} \Omega_{2}}$, the distribution of $Z=\Omega_{2}+\Omega_{1}$ is log-normal with parameters given by

$$
\begin{aligned}
\mu_{\mathrm{Z}}= & \mu_{\Omega_{1}}+G_{1}\left(\mu_{\mathrm{Y}}, \sigma_{\mathrm{Y}}\right) \\
\sigma_{\mathrm{Z}}^{2}= & \sigma_{\Omega_{1}}^{2}-G_{1}^{2}\left(\mu_{\mathrm{Y}}, \sigma_{\mathrm{Y}}\right)+G_{2}\left(\mu_{\mathrm{Y}}, \sigma_{\mathrm{Y}}\right) \\
& +2 \frac{\sigma_{\Omega_{1}}}{\sigma_{\mathrm{Y}}^{2}}\left(\rho_{\Omega_{1} \Omega_{2}} \sigma_{\Omega_{2}}-\sigma_{\Omega_{1}}\right) G_{3}\left(\mu_{\mathrm{Y}}, \sigma_{\mathrm{Y}}\right),
\end{aligned}
$$

where $Y=\Omega_{2}-\Omega_{1}$, and where $G_{1}(\cdot), G_{2}(\cdot)$ and $G_{3}(\cdot)$ are functions obtained by a numerical polynomial approximations [34].

It is known that the accuracy of the SY approximation tends to degrade as the number of summands increases; the number of transmitters in a SFN can be very large, specially if low power transmitters are in use. To palliate this, we use exact closed-form expressions for $G_{1}(\cdot), G_{2}(\cdot)$ and $G_{3}(\cdot)$ from [35]. These expressions are based on the $\operatorname{erfcx}(\cdot)$ function and prevent numerical instability and successive error accumulation in (3). On the other hand, the accuracy of the SY approximation may also worsen when the summands have different $\mu_{\Omega_{i}}$ and $\sigma_{\Omega_{i}}$ values, or when $\sigma$ is large [33,34]. However, we can confirm that the SY approximation is sufficiently accurate for this problem. This is mainly because in fixed television reception, the assumed value of $\sigma$ is small (5.5dB) [20] and the target outage levels are around $\left(1-q_{\mathrm{thr}}\right) \geq 5 \%$, far from where the SY method starts to degrade [33].

To evaluate (1), we follow the reformulation in [36] that avoids the summation of a log-normal RV with a truly deterministic variable: it rewrites (1) as

$$
\begin{aligned}
q & =\operatorname{Pr}\left\{1 \geq \frac{\gamma_{\min } N_{0}}{S}+\frac{\gamma_{\min } U}{S}\right\} \\
& =\operatorname{Pr}\{1 \geq A+B\} \\
& =\operatorname{Pr}\{1 \geq X\} \\
& =\frac{1}{2} \operatorname{erfc}\left(\frac{1}{\sqrt{2}} \frac{\mu_{X}}{\sigma_{X}}\right),
\end{aligned}
$$

where $\operatorname{erfc}()$ is the Gaussian complementary error function, and where $A, B$ are log-normal RVs. Here, $A$ is the ratio between the deterministic value $\gamma_{\min } N_{0}$ and log-normal RV $S$, whereas $B$ is the ratio between log-normal $\mathrm{RVs} \gamma_{\min } U$ and
$S$. In order to obtain the parameters $\mu_{X}$ and $\sigma_{X}$ that define the distribution of the resulting log-normal $\mathrm{RV} X=A+B$, we apply the SY method for the sum of two correlated log-normal random variables $A$ and $B$, which are defined by

$$
\begin{aligned}
\mu_{\mathrm{A}} & =10 \log _{10}\left(\gamma_{\min } \cdot N_{0}\right)-\mu_{\mathrm{S}} \\
\sigma_{\mathrm{A}} & =\sigma_{\mathrm{S}} \\
\mu_{\mathrm{B}} & =10 \log _{10}\left(\gamma_{\min }\right)+\mu_{\mathrm{U}}-\mu_{\mathrm{S}} \\
\sigma_{\mathrm{B}} & =\sqrt{\sigma_{\mathrm{S}}^{2}-2 \rho_{\mathrm{SU}} \sigma_{\mathrm{S}} \sigma_{\mathrm{U}}+\sigma_{\mathrm{U}}^{2}} \\
\rho_{\mathrm{AB}} & =\frac{\sigma_{\mathrm{S}}-\rho_{\mathrm{SU}} \sigma_{\mathrm{U}}}{\sqrt{\sigma_{\mathrm{S}}^{2}+\sigma_{\mathrm{U}}^{2}-2 \rho_{\mathrm{SU}} \sigma_{\mathrm{S}} \sigma_{\mathrm{U}}}} .
\end{aligned}
$$

Note that the difference with respect to [36], which assumes uncorrelated signals, is in the expressions for $\rho_{\mathrm{AB}}$ and $\sigma_{\mathrm{B}}$ given $\rho_{\mathrm{SU}}>0$. To find this value, we follow the method in [32], where $\rho_{\mathrm{SU}}$ is estimated through

$$
\rho_{\mathrm{SU}}=\frac{2\left[\log (v) / \xi^{2}-\xi\left(\mu_{\mathrm{S}}+\mu_{\mathrm{U}}\right)\right]-\left(\sigma_{\mathrm{S}}^{2}+\sigma_{\mathrm{U}}^{2}\right)}{2 \sigma_{\mathrm{S}} \sigma_{\mathrm{U}}},
$$

where $v$ is given by

$$
\begin{aligned}
v & =E(S U)=E\left[e^{S_{[\mathrm{dBB}]} / \xi} \cdot e^{U_{[\mathrm{dB}]} / \xi}\right] \\
& =\sum_{j=1}^{J} \sum_{k=1}^{K} E\left[e^{S_{j[\mathrm{dBB}]} / \xi+U_{k[\mathrm{dBB}]} / \xi}\right] \\
& =\sum_{j=1}^{J} \sum_{k=1}^{K} e^{\left(\xi \mu_{\mathrm{s}_{j}}+\xi \mu_{\mathrm{U}_{k}}+\rho \sigma^{2}\right) / \xi^{2}}
\end{aligned}
$$

and where vectors $\mu_{\mathrm{S}}=\left[\mu_{\mathrm{S}_{1}}, \ldots, \mu_{\mathrm{S}_{J}}\right]$ and $\boldsymbol{\mu}_{\mathrm{U}}=$ $\left[\mu_{\mathrm{U}_{1}}, \ldots, \mu_{\mathrm{U}_{K}}\right]$ contain the median received signal from each $j$ th and $k$ th transmitter.

\section{Spectrum Efficiency Calculation}

To obtain the maximum achievable efficiency of spectrum use, which is a function of both the size of the broadcasters' coverage area and the distance separating them, we pack broadcasters in a regular hexagonal tessellation to achieve the highest average density of broadcasters on a per area basis. See Fig. 1 and Fig. 2. We consider a statistical path loss model (instead of a terrain-aware model), thus median path loss depends only on the distance from each transmitter.

For a traditional broadcaster, we make the approximation that its interference-limited coverage area is sufficiently well represented by a circle centered at the transmitter, with radius $R_{\text {trad }}$ equal to the distance between the transmitter and the nearest point on the edge of the coverage area. At least for the range of numerical values considered in this paper, we observe little difference between the distance to the nearest point at the edge of coverage and the distance to the furthest point. Under this assumption, we define $C_{\text {trad }}$ as the minimum distance between coverage areas of two traditional broadcasters.

For the case of an SFN, as further discussed in III-D, we arrange transmitters in a regular hexagonal tiling that forms a reference hexagonal network $[26,37]$ and we make the approximation that a coverage area can be reasonably represented by 


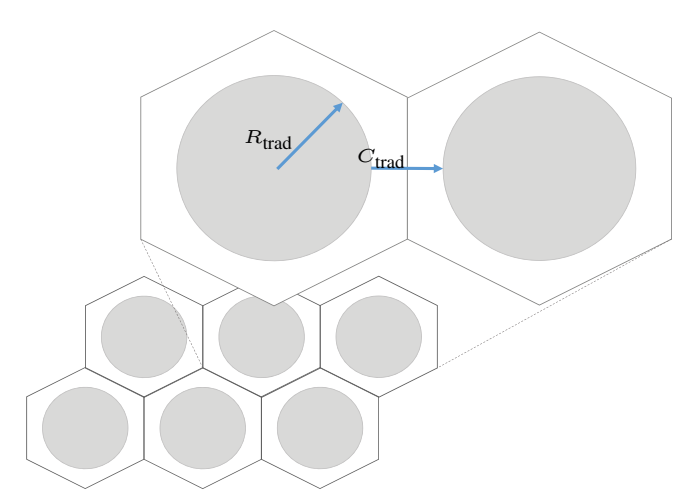

Fig. 1. Hexagonal packing of co-channel traditional broadcasters.

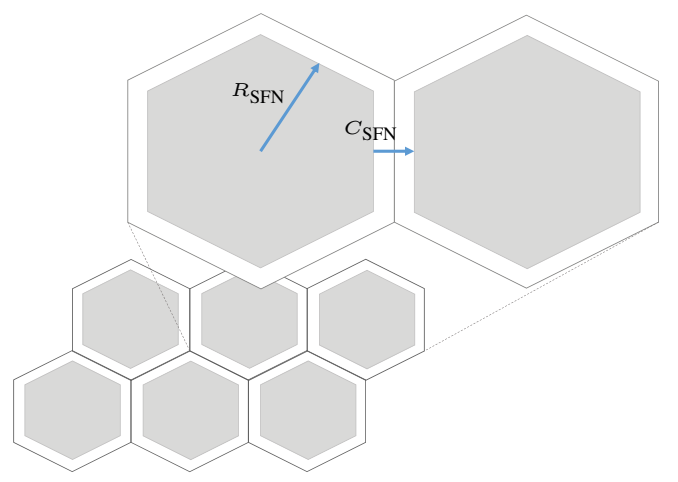

Fig. 2. Hexagonal packing of co-channel SFN broadcasters.

a regular hexagon of side $R_{\mathrm{SFN}}$, while considering a constant separation distance $C_{\mathrm{SFN}}$ between two SFNs coverage areas. As discussed further in III-D, because the edge of coverage is not a perfect line, the distance between two coverage areas is not exactly the same at all points. Thus, we calculate a value for separation distance that is roughly the average. For the range of numerical values used in this paper, we observe that this is a valid approximation.

From the above, we estimate the maximum fraction of area that can be covered by traditional broadcasters or SFNs divided by the area of their respective hexagonal tile in the lattice, which is given by

$$
\begin{aligned}
\eta_{\mathrm{trad}} & =\frac{R_{\mathrm{trad}}^{2}}{\left(R_{\mathrm{trad}}+0.5 C_{\mathrm{trad}}\right)^{2}} \cdot \frac{\pi}{2 \sqrt{3}} \\
\eta_{\mathrm{SFN}} & =\frac{R_{\mathrm{SFN}}^{2}}{\left(R_{\mathrm{SFN}}+0.5 C_{\mathrm{SFN}}\right)^{2}}
\end{aligned}
$$

\section{Calculation of Service Area Separation Distances}

As spectrum efficiency is defined as a function of the separation distance between coverage areas, we develop analytical expressions for their calculation. We divide the analysis in two pieces: traditional broadcasters and SFNs.

1) Traditional Broadcasting: By definition, coverage probability needs to be equal to the minimum threshold $q_{\text {thr }}$ for a point located at the edge of coverage. To obtain $C_{\text {trad }}$, we

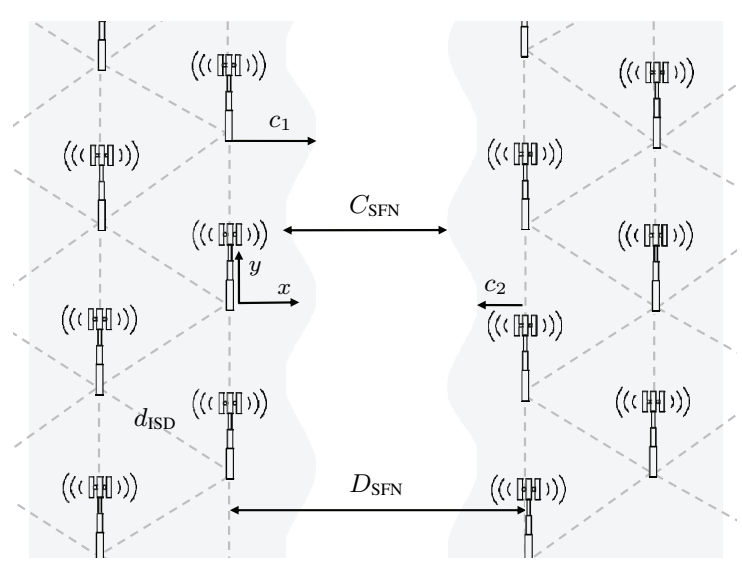

Fig. 3. Hexagonal lattice model for reference SFN deployment.

consider only the three closest interferers, as farther signals are at much lower levels to be considered relevant. Following Fig. 1, we calculate $C_{\text {trad }}$ along the line between the desired transmitter and its closest interferer, which corresponds to the minimum distance between two points in opposing coverage areas. In such a case, the receiver is located at a distance $R_{\text {trad }}$ from the desired transmitter, and at distances $d_{1}=R_{\text {trad }}+C_{\text {trad }}$ and $d_{2}=d_{3}=\left[3 R_{\text {trad }}^{2}+3 R_{\text {trad }} C_{\text {trad }}+C_{\text {trad }}^{2}\right]^{1 / 2}$ from the closest and the two second closest interferers respectively, which are oriented at $0^{\circ}$ and $\pm 60^{\circ}$ from the desired transmitter.

From (1), we express $q$ as an explicit function of $\gamma_{\min }$ and log-normal parameters $\mu_{\mathrm{S}}$ and $\mu_{\mathrm{U}}$. Hence, the relationship between transmit power $P$ and the separation distance between coverage areas $C_{\text {trad }}$ can be formulated as

$$
\begin{aligned}
& C_{\text {trad }}: q\left(\boldsymbol{\mu}_{\mathrm{S}}, \boldsymbol{\mu}_{\mathrm{U}} ; \gamma_{\text {min }}\right)=q_{\mathrm{thr}} \\
& \text { where } \mu_{\mathrm{S}_{1}}=P+g_{0}-L\left(R_{\text {trad }} ; h\right) \\
& \text { and } \mu_{\mathrm{U}_{k}}=P+g_{0}-L\left(d_{k} ; h\right)-\psi \\
& \quad \text { for } k=1,2,3
\end{aligned}
$$

where $g_{0}$ is the maximum gain of the directional reference antenna, and the value $\psi$ is its front-to-back (FB) ratio.

We parameterize transmit power as $P=P_{0}+\Delta P$, where $P_{0}$ is the transmit power required for a coverage area of size $R_{\text {trad }}$ in the noise-limited case, so $\Delta P>0$ is the interference margin that will allow obtaining smaller separation distances by allowing additional co-channel interference at the edge of coverage. Thus, to understand how $C_{\text {trad }}$ scales with $\Delta P$ for any given $R_{\text {trad }}$.

2) Single Frequency Networks: For SFNs, we assume they are deployed in a hexagonal lattice configuration, where transmitters are placed at a constant inter-site distance $d_{\text {ISD }}$, forming an hexagonal reference network of $N$ tiers surrounding a central transmitter site. We assume all transmitters to be identical, i.e. same antenna height $h$ and same transmit power $P$, and their antennas are assumed omni-directional in azimuth.

To obtain the minimum separation distance between two SFNs coverage areas, $C_{\mathrm{SFN}}$, we place two hexagonal SFNs facing each other as shown in Fig. 3. Given our definition of 


$$
\mathbf{d}\left(x, y ; r_{\mathrm{ISD}}, N\right)= \begin{cases}\sqrt{\left(x+3 r_{\mathrm{ISD}} \cdot m\right)^{2}+\left(y+\sqrt{3} r_{\mathrm{ISD}} \cdot n\right)^{2}} & m=0, \ldots, N / 2 \\ & n=-N / 2-m, \ldots, N / 2+m \\ \sqrt{\left(x+3 r_{\mathrm{ISD}}[m+1 / 2]\right)^{2}+\left(y+\sqrt{3} r_{\mathrm{ISD}}[n+|n| / 2 n]\right)^{2}} & m=0, \ldots, N / 2-1 \\ & n=-N / 2-m, \ldots, N / 2+m\end{cases}
$$

coverage stated in III-A, we have two thresholds $q_{\mathrm{thr}}^{\prime}>q_{\mathrm{thr}}$, which means a contiguous coverage area is formed by an inner region where coverage prob $q \geq q_{\mathrm{thr}}^{\prime}$ that is surrounded by an outer region where coverage probability is between $q_{\mathrm{thr}}$ and $q_{\mathrm{thr}}^{\prime}$. If we decrease the distance between the infrastructure for two SFNs, $D_{\mathrm{SFN}}$, then the coverage beyond the last tier of transmitters will shrink due to interference from the opposite SFN but it will also reduce $C_{\mathrm{SFN}}$. The coverage beyond the last tier of transmitters is represented by $c_{1}$ and $c_{2}$, which are measured along $x=0$ and $x=d_{\mathrm{ISD}} / 2$, where the origin of coordinates $x$ and $y$ is defined at the position of the transmitter located at the midpoint of the SFN's hexagonal side over the last tier (see Fig. 3). We observe that $C_{\mathrm{SFN}}$ decreases monotonically with respect to $D_{\mathrm{SFN}}$. As this occurs, there will be a point where further decreasing $D_{\mathrm{SFN}}$ will eventually cause a hole in coverage within the inner area where $q \geq q_{\mathrm{thr}}^{\prime}$ : we want SFNs as close together as possible without this to occur, i.e. without breaching our definition of coverage (note that for this to happen, the ISD value for each SFN has to be $d_{\mathrm{ISD}}<d_{\mathrm{ISD}_{\mathrm{NL}}}$, where $d_{\mathrm{ISD}_{\mathrm{NL}}}$ is the maximum ISD in the noise-limited case).

In general, separation distance $C_{\mathrm{SFN}}=D_{\mathrm{SFN}}-c_{1}-c_{2}$ is a function of both $D_{\mathrm{SFN}}$ and $d_{\mathrm{ISD}}$. As distance $C_{\mathrm{SFN}}$ monotonically decreases with respect to distance $D_{\mathrm{SFN}}$ when holding infrastructure constant, minimizing $C_{\mathrm{SFN}}$ is equivalent to minimizing $D_{\mathrm{SFN}}$. Hence, we characterize $C_{\mathrm{SFN}}$ through the following steps:

- We set each SFN's infrastructure by selecting an ISD value $d_{\mathrm{ISD}}<d_{\mathrm{ISD}_{\mathrm{NL}}}$ and by selecting a number of transmitter tiers given by $N$.

- We obtain the minimum $D_{\mathrm{SFN}}$ by setting the coverage probability value of the location where the hole in coverage occurs, which we define as $q_{\min }$, at the value equal to the minimum threshold $q_{\mathrm{thr}}^{\prime}$.

- To find the location of the coverage hole, we use well known constrained gradient-based numerical methods since the coverage probability surface is continuous and well-behaved: we minimize $q$ over the triangular region formed by the two closest transmitters beneath the transmitter located at $x=0$ and $y=0$. For the parameter values considered in this paper, we find that regardless of $D_{\mathrm{SFN}}$, the local minimum where $q_{\mathrm{min}}$ occurs is located approximately at $(x, y)=\left(-r_{\mathrm{ISD}}, 0\right)\left(r_{\mathrm{ISD}}=d_{\mathrm{ISD}} / \sqrt{3}\right)$, which is the equidistant point between the three transmitters.

- As $D_{\mathrm{SFN}}=C_{\mathrm{SFN}}+c_{1}+c_{2}$, we then solve for $c_{1}$ and $c_{2}$ which are both a function of ISD, $D_{\mathrm{SFN}}$ and $q_{\mathrm{thr}}<q_{\mathrm{thr}}^{\prime}$.
From the stated above, the relationship between $D_{\mathrm{SFN}}, d_{\mathrm{ISD}}$ and $q_{\mathrm{thr}}^{\prime}$ can be expressed as

$$
D_{\mathrm{SFN}}: \min _{x, y} q\left(\boldsymbol{\mu}_{\mathrm{S}}, \boldsymbol{\mu}_{\mathrm{U}} ; \gamma_{\mathrm{min}}\right)=q_{\mathrm{thr}}^{\prime}
$$

where $\mu_{\mathrm{S}_{j}}=P+g_{0}^{\prime}-L\left(d_{j}(x, y)\right)$

$$
\begin{gathered}
\mu_{\mathrm{U}_{k}}=P+g_{0}^{\prime}-L\left(d_{k}\left(D_{\mathrm{SFN}}-x, y+d_{\mathrm{ISD}} / 2\right)\right)[\mathrm{dB}] \\
\text { for } j=1, \ldots, J \text { and } k=1, \ldots, K
\end{gathered}
$$

where $g_{0}^{\prime}$ is the gain of the omni-directional reference antenna, and where $d_{j}(x, y) \in\left[d_{1}, \cdots, d_{J}\right]$ and $d_{k}(x, y) \in\left[d_{1}, \cdots, d_{K}\right]$ are distances between the receiver and each SFN transmitters. The vector containing these distances, $\mathbf{d}(x, y)$, can be summarized in (11) as a function of $r_{\text {ISD }}$ and $N$.

In a similar way, the expression for $c_{1}$ is

$$
\begin{array}{rr}
c_{1}: & q\left(\boldsymbol{\mu}_{\mathrm{S}}, \boldsymbol{\mu}_{\mathrm{U}} ; \gamma_{\min }\right)-q_{\mathrm{thr}}=0 \\
\mu_{\mathrm{S}_{j}}=P+g_{0}^{\prime}-L\left(d_{j}\left(c_{1}, 0\right)\right) & {[\mathrm{dB}]} \\
\mu_{\mathrm{U}_{k}}=P+g_{0}^{\prime}-L\left(d_{k}\left(D_{\mathrm{SFN}}-c_{1}, d_{\mathrm{ISD}} / 2\right)\right) & {[\mathrm{dB}]} \\
& \text { for } j=1, \ldots, J \text { and } k=1, \ldots, K
\end{array}
$$

and similarly, $c_{2}$ can be expressed as

$$
\begin{aligned}
c_{2}: & q\left(\boldsymbol{\mu}_{\mathrm{S}}, \boldsymbol{\mu}_{\mathrm{U}} ; \gamma_{\min }\right)-q_{\mathrm{thr}}=0 \\
\mu_{\mathrm{S}_{j}}=P+g_{0}^{\prime}-L\left(d_{j}\left(c_{2}, d_{\mathrm{ISD}} / 2\right)\right) & {[\mathrm{dB}] } \\
\mu_{\mathrm{U}_{k}}=P+g_{0}^{\prime}-L\left(d_{k}\left(D_{\mathrm{SFN}}-c_{2}, 0\right)\right) & {[\mathrm{dB}] } \\
& \quad \text { for } j=1, \ldots, J \text { and } k=1, \ldots, K
\end{aligned}
$$

With this, the size of a SFN coverage area can be approximated by

$$
R_{\mathrm{SFN}}=\frac{\sqrt{3}}{2} d_{\mathrm{ISD}} \cdot N+\frac{1}{2}\left(c_{1}+c_{2}\right)
$$

for a total number of transmitter sites $N_{\mathrm{SFN}}=1+6 \sum_{i}^{N} i$.

Note that although neither (12)-(14) are explicit equations in their variable of interest, we can nevertheless evaluate these expressions by using common root-finding algorithms, taking advantage they all represent monotonic relationships $\left(q_{\min }\right.$ over $D_{\mathrm{SFN}}$ and $C_{\mathrm{SFN}}$ over $\left.D_{\mathrm{SFN}}\right)$. On the other hand, to obtain a continuous relationship between $C_{\mathrm{SFN}}$ for a given value of $R_{\mathrm{SFN}}$, we interpolate the results obtained for $C_{\mathrm{SFN}}$ as a function of ISD for different values of $N$. This, as $R_{\mathrm{SFN}}$ will not likely coincide with an integer multiple of $d_{\mathrm{ISD}}$.

\section{E. Cost Calculations}

In III-D, we obtain for traditional broadcasting the transmit power level $P=P_{0}+\Delta P$ for a given coverage area size. Hence, in terms of the cost per one traditional broadcaster, we consider a transmitter operating 24 hours, 365 days per 
year, consuming a power of $P / \eta_{\mathrm{TX}}$ watts at a cost of $c_{k w-h}$ dollars per $\mathrm{kW}$ per hour, where $\eta_{\mathrm{TX}}$ is the power amplifier efficiency of the transmitter. For the case of SFNs, in III-D we obtain the approximate number of transmitters $N_{\mathrm{SFN}}$ for a given service area size given by $R_{\mathrm{SFN}}$, so we simply use an estimate of the NPV of the cost of building and operating one SFN transmitter, $\mathrm{NPV}_{\text {site. }}$ In this estimate we consider only the costs associated with equipment and its installation, and operation and maintenance of each site (energy included), but not other costs in the programming distribution chain.

Summarizing, the NPV of the cost per broadcaster, for both traditional and SFN, is given by

$$
\begin{aligned}
\mathrm{NPV}_{\text {trad }} & =\sum_{t=1}^{N_{\text {per }}} \frac{P_{[\mathrm{kW}]} \cdot 365 \cdot 24 \cdot c_{\mathrm{kW}-\mathrm{h}}}{(1+i)^{t}} \cdot \frac{1}{\eta_{\mathrm{TX}}} \\
\mathrm{NPV}_{\mathrm{SFN}} & =N_{\mathrm{SFN}} \cdot \mathrm{NPV}_{\text {site }}
\end{aligned}
$$

where $i$ is the annual discount rate, and $N_{\text {per }}$ is the evaluation period in years.

\section{MOdel ApPLiCATION}

\section{A. Numerical Assumptions}

In our assumptions we use the link budget parameters for coverage calculations in current U.S. television spectrum policy [27]. We consider a carrier frequency of $615 \mathrm{MHz}$, as it is representative of the UHF band, which is part of the frequency bands already targeted to be reallocated to mobile broadband use in the future. As recommended in [27], we assume noise no other than thermal; $N_{0}=k T B+\mathrm{NF}$, where $k T$ is the background noise spectral density of the receiver, $B$ is the equivalent noise bandwidth of the receiver, and NF corresponds to the receiver's noise figure (in general, our results are independent of $B$ unless stated otherwise). We assume a probability threshold $q_{\mathrm{thr}}=50 \%$ for the edge of coverage. Inside SFNs, we consider $q_{\mathrm{thr}}^{\prime}=95 \%$ which represents a threshold for high-quality coverage [20,29]. For traditional broadcasting, we consider an antenna height of $300 \mathrm{~m}$ which is typical for a TV tower in Europe [20], and it represents the median height for a TV tower in the U.S. [38]. For SFNs, we consider an antenna height of $30 \mathrm{~m}$ which is the typical height of a cellular transmitter $[3,30]$, whereas for transmit power, we have considered a range from $43 \mathrm{dBm}$ to $52 \mathrm{dBm}$ over $6 \mathrm{MHz}$, which is in line with both typical values reported for LPLT SFNs $[26,39]$ and with likely non-ionizing radiation limits [3]. A summary can be found in Table I.

For the directional reference antenna, we consider the ITU-R BT.419-3 recommendation [28], while for the omnidirectional reference antenna, we consider a $g_{0}^{\prime}=5 \mathrm{dBi}$ gain as for a $1.25 \lambda$ dipole antenna. For the path loss function $L($ ), we use the ITU-R P.1546-5 propagation model [40] considering $90 \%$ and $10 \%$ time availability for wanted signal and interference respectively [27], regardless of transmission architecture.

For log-normal shadowing, we assume $\sigma=5.5 \mathrm{~dB}$ [40]. In terms of correlation, we follow $3 \mathrm{GPP}$ considering a constant value of $\rho=0.5$ [30]. This recommendation can be readily
TABLE I

Link Budget Parameters - Baseline CASE

\begin{tabular}{lcc}
\hline Parameter & & Value \\
\hline Carrier Frequency & $f$ & $615 \mathrm{MHz}$ \\
Minimum SINR & $\gamma_{\min }$ & $15 \mathrm{~dB}$ \\
Thermal Noise Spectral Density & $k T$ & $-174 \mathrm{dBm} / \mathrm{Hz}$ \\
Receiver Noise Figure & $\mathrm{NF}$ & $7 \mathrm{~dB}$ \\
Downlead line loss & $L_{\mathrm{line}}$ & $4 \mathrm{~dB}$ \\
Shadowing Standard Deviation & $\sigma$ & $5.5 \mathrm{~dB}$ \\
Edge of Coverage probability threshold & $q_{\mathrm{thr}}$ & $50 \%$ \\
Inner Coverage probability threshold & $q_{\mathrm{thr}}^{\prime}$ & $95 \%$ \\
\hline Traditional Broadcasting: & & \\
Antenna height & $h$ & $300 \mathrm{~m}$ \\
Directive antenna gain & $g_{0}$ & $12 \mathrm{dBi}$ \\
Directive antenna FB ratio & $\psi$ & $14 \mathrm{~dB}$ \\
\hline Single Frequency Networks: & & \\
Antenna height & $h_{i}$ & $30 \mathrm{~m}$ \\
Omni-directional antenna gain & $g_{0}^{\prime}$ & $5 \mathrm{dBi}$ \\
Transmit power spectral density & $P_{i}$ & $52 \mathrm{dBm} / 6 \mathrm{MHz}$ \\
\hline
\end{tabular}

applied to the case of SFNs due to its similarity with cellular networks. For the case of traditional broadcasting, we have found negligible numerical differences between considering either $\rho=0.5$, or considering $\rho=0$ as typically assumed. In our case, given that our coverage definition considers $q_{\mathrm{thr}}=$ $50 \%$, the potential effect of $\rho$ is canceled out; for higher values of $q_{\text {thr }}$ we might found that the $\rho=0$ assumption is quite pessimistic, leading to larger separation distances.

In terms of cost, for traditional broadcasting we assume $\eta_{\mathrm{TX}}=20 \%$, as the approximate power consumption for a state-of-the-art DVB-T transmitter of the year 2010 [11] which we assume representative of what can be found in the field today. We assume $c_{\mathrm{kW}-\mathrm{h}}=\$ 0.12$, which is close to the average energy cost for commercial/industry use in the U.S. [41]. Also, we consider $N_{\text {per }}=20$ years and $i=7 \%$, which is the recommended real interest rate of the U.S. Office of Management and Budget for cost-effectiveness analysis purposes [42]. Hence, we obtain $\$ 5,250$ per $\mathrm{kW}$ transmitted over the air per year, equivalent to a cost of $\$ 55,000$ per $\mathrm{kW}$ in present value over the evaluation period.

On the other hand, for SFNs we assume $\mathrm{NPV}_{\text {site }}=$ $\$ 650,000$, which is in line with values reported in [43] when considering $i=7 \%$ and $N_{\text {per }}=20$ years; we have assumed that the potential cost of building and operating one SFN site is similar to those of sites in cellular networks [3].

\section{B. Numerical Results}

In this section, we first present the spectrum efficiency results for each transmission alternative, before use them to analyze the cost-effectiveness of each strategy.

1) Spectrum Efficiency in Traditional Broadcasting: For traditional broadcasting, Fig. 4 shows $C_{\text {trad }}$ as a function of the interference margin $\Delta P$, with the required service area radius $R_{\text {trad }}$ as a parameter. For any service area size, increasing transmit power always reduces separation distance between coverage areas, but this exhibits diminishing returns. Separation distances are much higher when interference margin 


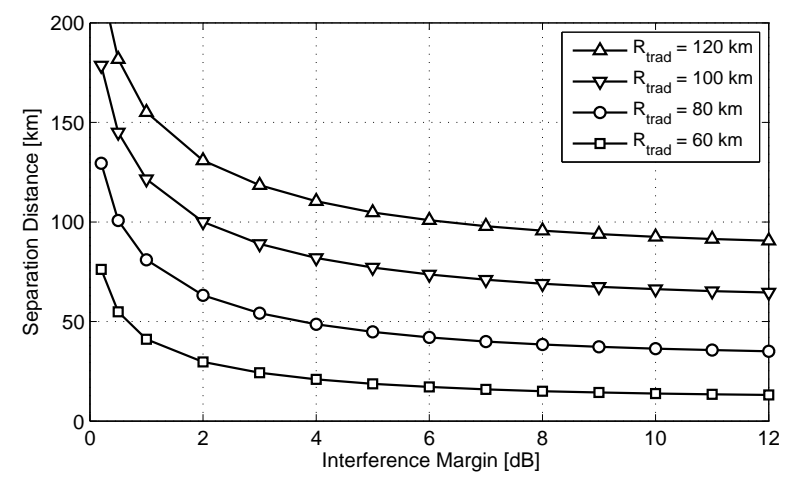

Fig. 4. Traditional broadcasting coverage area separation distance $C_{\text {trad }}$ as a function of interference margin $\Delta P$, with service area radius $R_{\text {trad }}$ as a parameter.

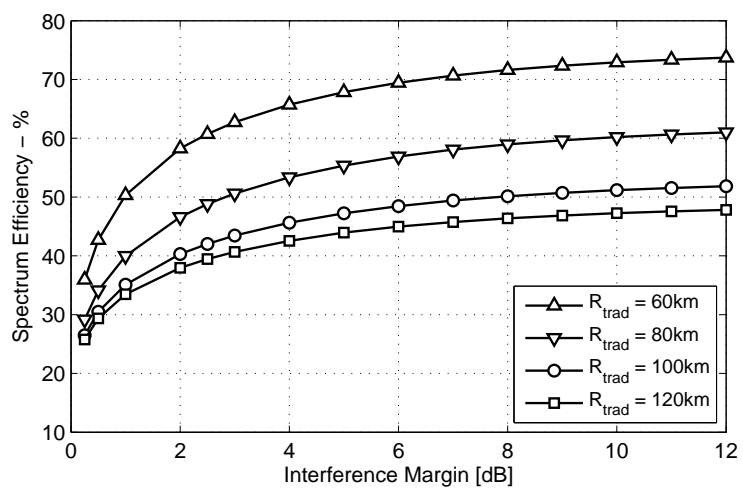

Fig. 5. Spectrum efficiency in traditional broadcasting as a function of interference margin $\Delta P$, with service area radius $R_{\text {trad }}$ as a parameter.

is below $1 \mathrm{~dB}$ (tolerable interference around $6 \mathrm{~dB}$ below noise level or less), which is approximately the current state in U.S. policy [27]. When tolerable interference is set at or above noise level, i.e. $\Delta P \geq 3 \mathrm{~dB}$, as in $\mathrm{G} 06$ [37], achievable separation distances are quite smaller. Diminishing returns occur at roughly the same point as measured in $\mathrm{dB}$, although an increase of $1 \mathrm{~dB}$ is much more costly if $P$ is high, i.e. if coverage area is large, than if $P$ is low. For subsequent results, we assume $\Delta P=1 \mathrm{~dB}$ as the baseline spectrum efficiency of a traditional broadcaster.

Fig. 4 shows that larger coverage areas require larger separation distances. However, as spectrum efficiency increases with coverage area and decreases with separation distance, we cannot if spectrum efficiency increases or decreases. Fig. 5 shows how power affects spectrum efficiency. Within this range, the larger the coverage area, the lower the spectrum efficiency, with all other parameters held constant. This suggests that it would be more efficient in terms of spectrum to provide TV service to a given area by using more smaller individual coverage areas rather than fewer larger ones, at least in a band consisting of TV broadcasters of similar size. In the U.S., current spectrum policy tends to favor large coverage areas over small ones.

2) Spectrum Efficiency in SFNs: Fig. 6 shows the relationship between $C_{\mathrm{SFN}}$ and ISD. As expected, SFN separation distances are smaller by approximately one order of magnitude compared to traditional broadcasters. Much like increasing transmit power for a traditional broadcaster as shown in Fig. 5, increasing cost by reducing $d_{\text {ISD }}$ in an SFN allows a broadcaster to tolerate more interference and thus reduce separation distances between coverage areas. Moreover, asymptotic performance is the same regardless of transmit power.

We also observe that, due to loss in macro diversity, higher correlation leads to a smaller ISD near the noise-limited regime as compared to when signals are uncorrelated, which is a simplifying and somewhat optimistic assumption. However, in the interference-limited regime, correlation slightly improves $C_{\text {SFN }}$ but does not significantly change the relationship between ISD and separation distance. These observations are consistent with results in [2].

A TV viewer using our reference antenna within coverage areas of multiple broadcasters will receive all of their signals, because our definition is based on an omni-directional receive antenna. This is reasonable because in the U.S. TV broadcasters choose where to locate and how much to cover. However, European TV spectrum policy is different and it dictates bordering area-based licenses (which also leads to a very different industry structure). In this regard, recent discussions have addressed the feasibility and cost of obtaining separation distances close to $0 \mathrm{~km}[3,21]$. To analyze this, we extend the calculations of $c_{1}$ and $c_{2}$ in (13) and (14) by using a directional antenna pointed in the best direction, which is roughly towards the closest transmitter. Results are shown in Fig. 7. Using a directional antenna with a FB ratio of $\psi=14 \mathrm{~dB}$ makes the asymptotic separation distance fall from about $8-10 \mathrm{~km}$ in the omni- case to about $2-3 \mathrm{~km}$. For higher values of FB, a $0 \mathrm{~km}$ separation distance is possible and without significantly reducing ISD.

3) Spectrum efficiency vs cost trade-off: In certain cases, low to moderate gains are desirable if the cost to achieve them is low, while in other cases, large gains in efficiency as those suggested by SFN performance, can be outweighed by its elevated cost. To analyze this, let's assume one wants to cover every location across a large area and that the coverage area per broadcaster is fixed. We consider a change in cost of transmission from an initial traditional broadcasting regime with spectrum efficiency $\eta_{1}$, to a more efficient regime $\eta_{2}>\eta_{1}$ due to either traditional broadcasting with increased transmit power or by switching to SFN transmission. Each channel that the network uses can cover a fraction $\eta$ of the area. As long as coverage areas in different channels do not overlap, the amount of spectrum needed per broadcast channel delivered to every point in a region is roughly $1 / \eta_{1}$. Hence, the average number of channels $N_{\text {ch }}$ that can be saved, by improving spectrum efficiency from $\eta_{1}$ to $\eta_{2}$ per channel of actual content delivered throughout the region is given by

$$
\Delta N_{\mathrm{ch}}=\frac{1}{\eta_{1}}-\frac{1}{\eta_{2}} .
$$

Fig. 8 shows how spectrum efficiency varies with total cost for a single broadcaster. For traditional broadcasting, 


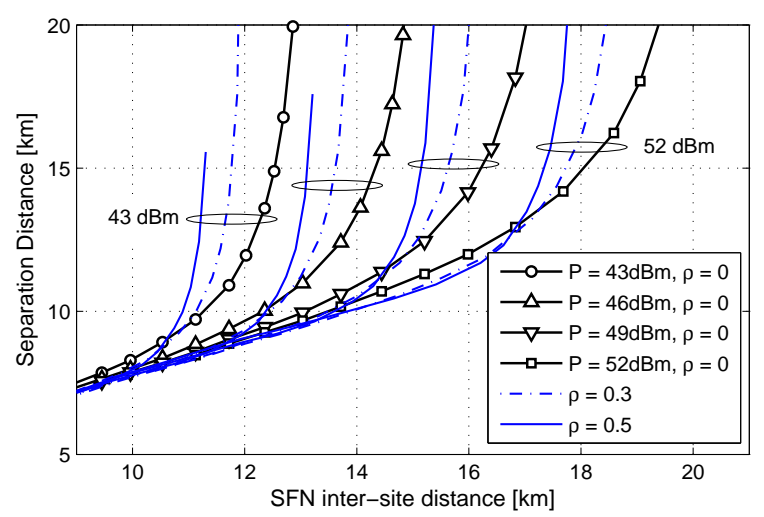

Fig. 6. Separation distance between SFN coverage areas as a function of ISD, for different values of transmit power $P$ and different values for correlation between signals $\rho$.

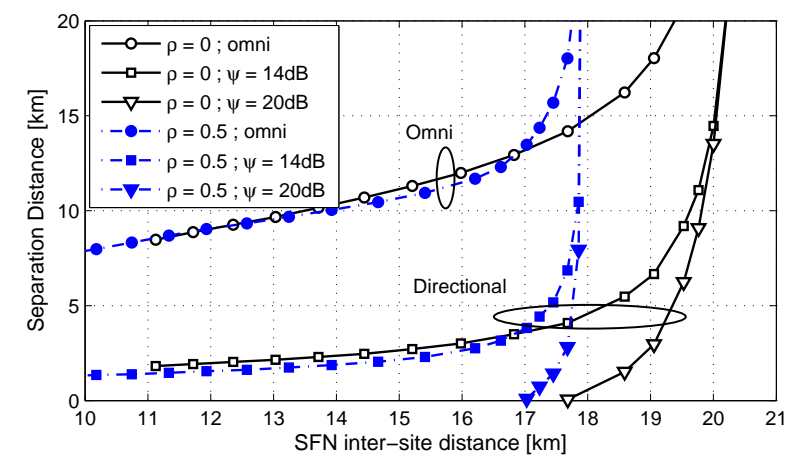

Fig. 7. Comparison between separation distances achievable with omnidirectional antennas and with directional antennas with $\psi=\{14 \mathrm{~dB}, 20 \mathrm{~dB}\}$.

we normalize the transmission cost with respect to the cost of a traditional broadcaster with baseline transmit power $(\Delta P=1 \mathrm{~dB})$, while for SFNs we normalize with respect to the cost of a close to noise-limited SFN. At least within the range of values we considered, for a given coverage area, an SFN has a greater spectrum efficiency than a traditional broadcaster, regardless of the ISD of the SFN or the transmit power of the traditional broadcaster. Nevertheless, increasing cost for a traditional broadcaster by a small percentage (by increasing transmit power) can significantly increase spectrum efficiency, whereas increasing cost for an SFN-based broadcaster (by decreasing ISD) produces a much smaller increase in spectrum efficiency. For example, consider a broadcaster with $R_{\text {trad }}=$ $100 \mathrm{~km}$. For an increase to $200 \%$ of the transmission cost, the absolute spectrum efficiency in traditional broadcasting increases from $\eta_{1}=35 \%$ to $\eta_{2}=46 \%$, equivalent to $24 \%$ less spectrum used, whereas the same increase in cost for an SFN design would lead to an increase in spectrum efficiency from $85 \%$ to $92 \%$ (less than $8 \%$ ).

Fig. 8 also shows that spectrum efficiency decreases with coverage area size in traditional broadcasting. For example, with $\Delta P=1 \mathrm{~dB}$, spectrum efficiency is $50 \%$ for a $60 \mathrm{~km}$ coverage area radius and $35 \%$ for a $120 \mathrm{~km}$ radius, so $60 \mathrm{~km}$ broadcasters can bring a given number of channels to everyone with approximately 30\% less spectrum (although four times as many broadcasters are needed). The spectrum efficiency

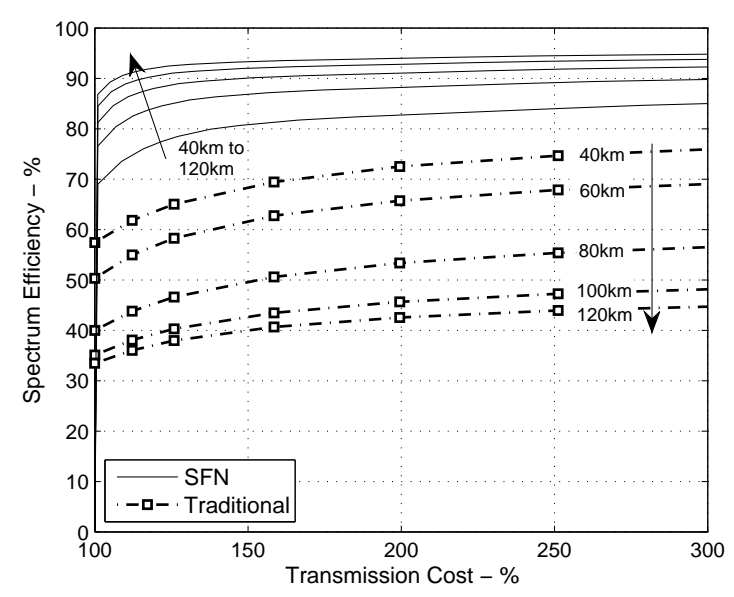

Fig. 8. Spectrum efficiency vs. transmission cost divided by baseline minimum cost, for different coverage area size radius $R_{\text {trad }}$. The arrow indicates the direction towards increasing radius.

advantage of smaller radius is even greater for larger transmit powers. In contrast, with SFNs, spectrum efficiency increases with coverage area. This is consistent with recent suggestions in spectrum policy discussions involving SFNs [3,29]. At least in U.S. policy, the size of coverage areas is a widelydiscussed issue all by itself, and it has big implications with respect to issues unrelated to spectrum including localism, civic discourse, emergency alerts, local vs national advertising, and more.

We now determine whether and when the benefit of increasing spectrum efficiency is worth the cost. In (18) we show the number of channels that can be saved per channel of actual content delivered throughout the region. The increase in cost per area of delivering that content is $\triangle \mathrm{NPV}=$ $\left(\mathrm{NPV}_{2}-\mathrm{NPV}_{1}\right) / A_{\mathrm{TV}}$ where $\mathrm{NPV}_{i}$ is the net present value of the per broadcaster cost of transmission in each regime $\eta_{i}$. Thus, we approximate the cost per sq-km per broadcast channel that can be freed by

$$
C_{\mathrm{MHz}-\mathrm{km}^{2}}=\frac{\left(\mathrm{NPV}_{2}-\mathrm{NPV}_{1}\right)}{A_{\mathrm{TV}}} \cdot \frac{1}{B \cdot \Delta N_{\mathrm{ch}}}
$$

The benefit of increasing spectrum efficiency depends on the value of spectrum. If the value of a $\mathrm{MHz}$-sq $\mathrm{km}$ is high enough, which often means if the population density is large enough, then the benefit will be worth the cost. To quantify when this will be the case, let us assume that value per MHz-POP is known, and that it is constant for all population densities. Hence, population density $\Pi_{\mathrm{POP}}=C_{\mathrm{MHz}-\mathrm{km}^{2}} / C_{\mathrm{MHz}-\mathrm{POP}}$. Thus, we would like to know the population density $\Pi_{\mathrm{POP}}$ at which either increasing transmit power in traditional broadcasting or switching to SFNs is worth the cost. For the value of $C_{\mathrm{MHz}-\mathrm{POP}}$ we look back at recent spectrum auctions in the U.S.; in the $700 \mathrm{MHz}$ auction in 2007 spectrum was sold for a national average of $\$ 1.28$ per MHz-POP [44], while the recent AWS3 auction in 2014 yielded an average of $\$ 2.71$ per $\mathrm{MHz}$ POP [44]. In this work, we consider $C_{\mathrm{MHz}-\mathrm{POP}}$ to be between $\$ 1$ and \$3, with $\$ 2$ per MHz-POP our baseline estimate. 


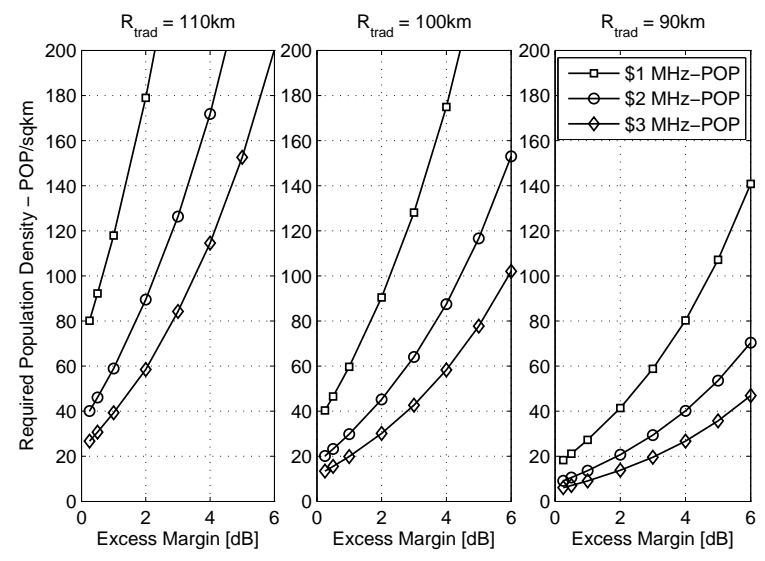

Fig. 9. Required population density for break-even vs. excess interference margin in traditional broadcasting, for different values of the cost per $\mathrm{MHz}-$ POP and for different values of service area radius.

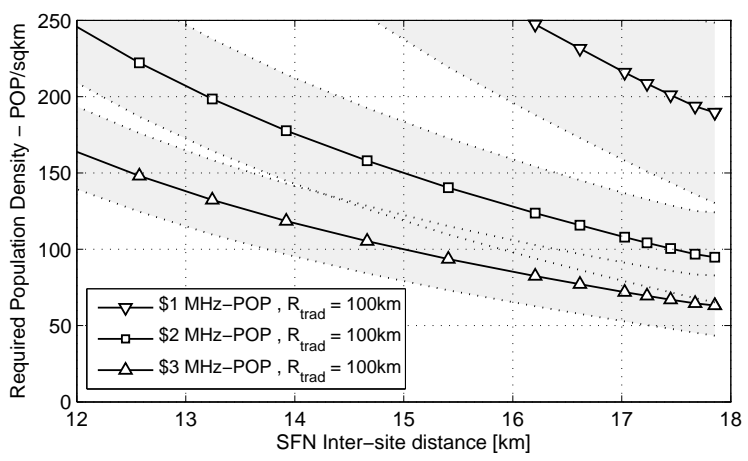

Fig. 10. Required population density for break-even vs. SFN inter-site distance, for different costs per MHz-POP and for different service area radius. Results obtained for $P=52 \mathrm{dBm} / 6 \mathrm{MHz}$

First, we analyze traditional broadcasting. In Fig. 9, we show the minimum required population density $\Pi_{\mathrm{POP}}$ at which the cost of increasing transmit power equals the value of spectrum freed as a function of the excess interference margin. We define the excess interference margin as the additional transmit power over $\Delta P=1 \mathrm{~dB}$. Since a typical full-power broadcaster in the U.S. covers approximately $100 \mathrm{~km}$ around the transmitter, we consider $R_{\text {trad }}$ being between $90 \mathrm{~km}$ and $110 \mathrm{~km}$. Fig. 9 shows that the break-even population densities are quite low, e.g. for a $100 \mathrm{~km}$ radius the break-even population density would be $30 \mathrm{POP} / \mathrm{sq} \mathrm{km}$ if spectrum is worth $\$ 2$ per MHz-POP, and $20 \mathrm{POP} / \mathrm{sq} \mathrm{km}$ if spectrum is worth $\$ 3$ per sq $\mathrm{km}$. This is small compared to many large areas in the U.S. The population density of the contiguous U.S. is 40 POP/sq km. 29 of 50 states have a population density above $30 \mathrm{POP} / \mathrm{sq} \mathrm{km}$, and 36 states have a population density above 20 [45]. This suggests that this strategy can be quite costeffective in a large fraction of the country.

Fig. 9 also shows that the larger the coverage area, the more costly it is to free spectrum this way. This translates to a higher required population density to break-even. Thus, the change from noise-limited to interference-limited coverage is even more cost-effective if coverage areas are smaller.

Fig. 10 shows the minimum population density $\Pi_{\mathrm{POP}}$ to break-even from a switchover to SFNs. We show in gray an uncertainty band on the coverage radius of the current traditional broadcasters, while the solid lines represent the results for $\$ 1, \$ 2$ and $\$ 3$ per MHz-POP for $R_{\text {trad }}=100 \mathrm{~km}$. If the smallest $\Pi_{\mathrm{POP}}$ were greater than the population density of New York City, then a switch to SFNs would always be a bad idea, but this is not the case. For a conservative estimate of $\$ 2$ in the value of the spectrum, the minimum $\Pi_{\mathrm{POP}}$ is in the order of $100 \mathrm{POP} / \mathrm{sq} \mathrm{km}$. At \$3 per MHz-POP, SFNs become cost-effective at a population density of just $67 \mathrm{POP} / \mathrm{sq} \mathrm{km}$. Our results show that this could be cost-effective in some parts of the U.S., but not in others. The U.S. East Coast corridor between Washington D.C. and Boston is a good example of a region that is likely to benefit from a transition to SFNs. It is a sufficiently large area and its average population density is at least $145 \mathrm{POP} / \mathrm{sq} \mathrm{km}$. We calculate this number from information available on a per state basis, which includes many rural areas. Thus, the actual population density along the coast is even higher.

In Fig. 11 we show the approximate fraction of spectrum that can be freed as a function of the incremental cost per sq $\mathrm{km}$ covered. A modest increase in transmission power can save over $30 \%$ of the spectrum, and transitioning to an SFN can save over $60 \%$, although an SFN is only worthwhile where spectrum is sufficiently valuable to justify the cost. Fig. 12 shows the approximate fraction of spectrum that can be freed with these two approaches when maximizing the value of spectrum freed minus the cost incurred. In this graph, we assume that the value of spectrum is $\$ 2$ per MHz-POP, which makes the amount of spectrum freed when maximizing value minus cost a function of population density. Fig. 12 also shows the amount of spectrum that can be freed as a function of population density with both approaches when the value of spectrum freed exactly equals the cost, so that any point in between these two curves yields a benefit minus cost that is superior to what we have with the noise-limited policy of today. The difference between benefit and cost achievable with SFNs exceeds that achievable with interferencelimited single-transmitter systems when the population density exceeds the vertical line just above $120 \mathrm{POP} / \mathrm{sq} \mathrm{km}$. As a result, if spectrum is worth $\$ 2$ per $\mathrm{MHz}-\mathrm{POP}$, then regions with population density above roughly $120 \mathrm{POP} / \mathrm{sq} \mathrm{km}$ should adopt SFNs, regions with population density between 30 and $120 \mathrm{POP} / \mathrm{sq} \mathrm{km}$ should use a traditional broadcast architecture but with increased transmit power, and regions with under 30 $\mathrm{POP} / \mathrm{sq} \mathrm{km}$ should maintain the current approach.

Unlike simply increasing transmit power of traditional broadcasters, switching to an SFN requires adoption of a new TV standard, such as ATSC 3.0 [8]. The results above do not include any costs incurred by consumers during such a transition, as would be appropriate if the new standard is to be adopted anyway. However, even if the change in standards occurs specifically to enable a transition to SFNs, this would produce only a modest change in our results. Every television that operates over-the-air (OTA) would require a converter. In general, the cost of converters per capita is the cost of a 


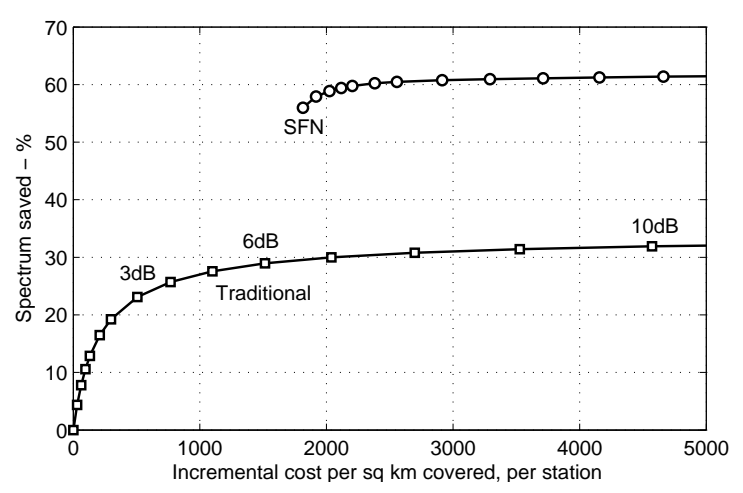

Fig. 11. Maximum percentage of spectrum savings vs. incremental cost per sq-km covered for each strategy for one station with $R_{\text {trad }}=100 \mathrm{~km}$.

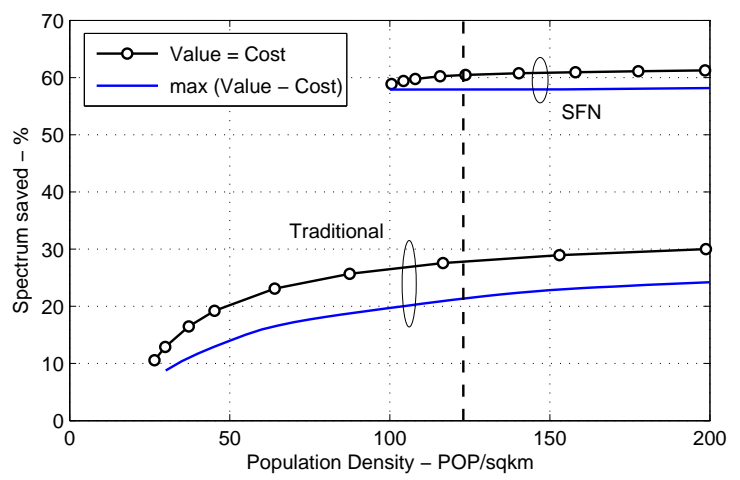

Fig. 12. Maximum percentage of spectrum savings vs population density for a) value of the spectrum freed equals cost, and b) maximum net benefit.

converter times number of TVs per capita times the fraction of TVs using OTA. In the U.S., there are roughly 1.1 TVs per capita, since the CEA estimates 2.9 TVs per household [46] and the U.S. Census indicates there are 2.63 people per household [45]. We will assume that roughly $13 \%$ of TVs receive OTA broadcasts, given that the National Association of Broadcasters (NAB) says it is $20 \%$ and the CEA says $7 \%$ [47]. It has been estimated that the cost of a converter will be around $\$ 100$ [48]. Thus, the cost to TV viewers is roughly $\$ 14$ per capita. In the U.S. today, $216 \mathrm{MHz}$ of UHF spectrum are used for television. This means that a switch to SFNs could free up roughly $130 \mathrm{MHz}$ of spectrum $(60 \%$ of $216 \mathrm{MHz})$ in the most densely populated parts of the country where broadcasters are most closely packed. Then, the cost of converters is just $\$ 0.11$ per MHz-POP, which is small compared to the baseline \$2 per $\mathrm{MHz}-\mathrm{POP}$ at break-even population density.

\section{CONCLUSiOns}

In this paper, we analyze two technical strategies that increase spectrum reuse for broadcast television while keeping the coverage area size and bandwidth per TV broadcaster largely unchanged. One is to boost the transmit power in traditional single-transmitter broadcasting and the other is to switch to SFNs. For both strategies, we characterize the tradeoff between spectrum efficiency and cost per area covered. With both strategies, we consider the possibility that additional interference is tolerated at the edge of coverage, which greatly reduces the separation distance between coverage areas and thus increases the reuse of the spectrum.

We develop a theoretical analytical method to calculate spectrum efficiency as a function of transmit power in a traditional architecture or as a function of ISD in SFNs, which are the variables that most affect cost in each case. We find that increasing transmit power for a traditional single-transmitter architecture can free up roughly $30 \%$ of the spectrum used for television, assuming that the stations are packed together as closely as possible both before and after the transition. Switching to an SFN can free up over $60 \%$ of the spectrum used for television. In the most densely populated parts of the U.S. where broadcasters are most closely packed, SFNs could free up to roughly $130 \mathrm{MHz}$ of UHF spectrum (60\% of 216 $\mathrm{MHz}$ ). This is also where spectrum is in greatest demand for other purposes such as broadband Internet access. It would be hard to find another change in spectrum usage that can come close to making that much prime spectrum in densely populated areas available for reallocation.

However, these increases in spectrum efficiency come at a cost that cannot be ignored. It is only worth adopting a new strategy for TV broadcasting if the value of the spectrum freed exceeds that cost. If spectrum is worth $\$ 2$ per $\mathrm{MHz}-$ $\mathrm{POP}$, then a region with $30 \mathrm{POP} / \mathrm{sq} \mathrm{km}$ or more would benefit from a switch to an interference-limited approach in traditional broadcasting rather than the nearly noise-limited approach dictated by today's spectrum policy. Much of the U.S. has a population density above this threshold, so it is likely that much of the U.S. would benefit. This approach could be adopted in concert with the repacking that is expected to follow the incentive auction for TV spectrum in 2016, and would have no apparent impact on consumers.

Some of the U.S. could benefit even more from a switch to SFNs. Because SFNs free up considerably more spectrum per area but are also more expensive, SFNs make sense only where population density is even higher. If spectrum is valued at \$2 per MHz-POP, then SFNs would be cost effective for population densities of roughly $120 \mathrm{POP} / \mathrm{sq} \mathrm{km}$ or more over an area large enough to include multiple broadcast markets. Although this paper does not explicitly address the case where parts of the U.S. adopt SFNs and other parts do not, we note that there are large regions where the population density is well over this threshold, including a large portion of the East Coast, where the use of SFNs is likely to be cost-effective.

Of course, even where overall benefits exceed overall costs, there is a risk that some parties may suffer from a change in policy and technology. In particular, this approach shifts costs onto broadcasters. Policies are needed to shift benefits to broadcasters as well, although these policies are outside the scope of this paper which focuses only on estimating overall costs and benefits.

Finally, it is important to note that spectrum efficiency depends on broadcaster service areas. Under the current approach of single-transmitter systems that are noise-limited, broadcasters with smaller coverage areas use spectrum more 
efficiently. For example, $4 n$ closely packed broadcasters with $60 \mathrm{~km}$ radius would cover the same total area as $n$ broadcasters with $120 \mathrm{~km}$ radius, but those with the $60 \mathrm{~km}$ radius could do so with roughly $30 \%$ less spectrum. The spectrum-efficiency advantages of smaller coverage area are even greater if transmit power is increased. Many spectrum policies give priority to broadcasters with large coverage areas, even though doing so implicitly favors less efficient use of spectrum. However, this is not the case if broadcasters switch to SFNs, because spectrum efficiency increases with coverage area for SFNs.

\section{ACKNOWLEDGMENT}

This material is based in part upon work supported by the National Science Foundation under Grant No. 1343359. Any opinions, findings, and conclusions or recommendations expressed in this material are those of the authors and do not necessarily reflect the views of the National Science Foundation.

The authors also want to thank the financial support of CONICYT Chile and the J. William Fulbright Scholarship Board.

\section{REFERENCES}

[1] J. Rebhan and J. Zander, "On the Outage Probability in Single Frequency Networks for Digital Broadcasting." IEEE Trans. Broadcast., vol. 39, no. 4, pp. 395 - 401, Dec. 1993.

[2] G. Malmgren, "On the Performance of Single Frequency Networks in Correlated Shadow Fading," vol. 43, no. 2, pp. 155-165, Jun. 1997.

[3] D. Lewin, P. Marks, Y. S. Chan, W. Webb, C. Chatzicharalampous and T. Jacks, "Final Report: Challenges and Opportunities of BroadcastBroadband Convergence and its Impact on Spectrum and Network Use. A Study prepared for the European Commission,” Dec. 2014.

[4] J. Zander and P. Mahonen, "Riding the Data Tsunami in the cloud: Myths and challenges in future wireless access." IEEE Commun. Mag., vol. 51, no. 3, pp. 145 - 151, Mar. 2013.

[5] CTIA and CEA Comments on NBP Public Notice \#26: Exploring a Path for Next Gen Television and Next Gen Wireless Broadband Spectrum, GN Docket No. 09-51, Dec. 22, 2009.

[6] Federal Communications Commission, "The National Broadband Plan Working Reports and Technical Papers," April 2010.

[7] Final Report on ATSC 3.0 Next Generation Broadcast Television, ATSC Planning Team 2, Sept. 2011.

[8] ATSC Technology Group 3, Call for Proposals For ATSC 3.0 Physical Layer: A Terrestrial Broadcast Standard, March 26, 2013.

[9] P. Lamy, "Report to the European Comission: Results of the work of the high level group on the future use of the UHF band (470-790 MHZ)," Sep. 2014.

[10] CEPT Task Group 6 (TG6), "ECC Report 224 Long Term Vision for the UHF broadcasting band," Nov. 2014

[11] J. Huschke, J. Sachs, K. Balachandran, and J. Karlsson, "Spectrum Requirements for TV Broadcast Services using Cellular Transmitters," in Proceedings of IEEE DySPAN, 2011.

[12] L. Shi, E. Obregon, K. W. Sung, J. Zander, and J. Bostrom, "CellTV - On the Benefit of TV Distribution over Cellular Networks: A Case Study," IEEE Trans. Broadcast., vol. 60, no. 1, pp. 73-84, Mar. 2014.

[13] L. Shi, K. W. Sung and J. Zander, "Spectrum Requirement for Cellular TV distribution in UHF Band from Urban to Rural Environment," in Proceedings of IEEE DySPAN, Apr. 2014.

[14] A. Mattsson, "Single Frequency Networks in DTV," IEEE Trans. Broadcast., vol. 51, no. 4, pp. 413-422, Dec. 2005.

[15] Association for Maximum Service Television and the National Association of Broadcasters, Reply Comments - NBP \#30: A National Broadband Plan for Our Future, GN Docket No. 09-51, Jan. 27, 2010.

[16] M. El-Hajjar and L. Hanzo, "A Survey of Digital Television Broadcast Transmission Techniques,” vol. 15, no. 4, pp. 1924-1949, Dec. 2013.
[17] 3GPP TS 25.346, Introduction of the Multimedia Broadcast Multicast Service (MBMS) in the Radio Access Network (Rel. 8), Mar. 2008.

[18] Digital Video Broadcasting (DVB); Implementation guidelines for a second generation digital terrestrial television broadcasting system (DVBT2), DVB Document A133 February 2012.

[19] ONE Media LLC, "NGBP Physical Layer Overview [Online]," in http://www.onemediallc.com/presentations, Sep. 2014.

[20] European Broadcasting Unit - Tech. Report 3348, "Frequency and Network Planning Aspects of DVB-T2", Geneva, May 2013.

[21] Qualcomm/ATDI, "Low Power Low Tower SFN networks: Interference Analysis [Online]," in http://www.atdi.com/lplt/, Oct 2014.

[22] P. Nygren, "High and Low Tower Broadcast Networks, Progira Report [Online]," in http://www.progira.com, Apr. 2014.

[23] R. Brugger and A. Schertz, "TV Distribution via Cellular Networks Part 1: Spectrum Consumption," in EBU Technical Report, May. 2014.

[24] _ , "TV Distribution via Cellular Networks Part 2: Cost Aspects," in EBU Technical Report, May. 2014.

[25] EBU Technical Report, "Delivery of Broadcast Content over LTE Networks," Jul. 2014.

[26] — "DVB-T2 Single Frequency Networks and Spectrum Efficiency," Nov. 2014.

[27] FCC OET Bulletin No. 69: Longley-Rice Methodology for Evaluating TV Coverage and Interference, Feb. 06, 2004.

[28] ITU-R, "Directivity and polarization discrimination of antennas in the reception of television broadcasting", Recommendation ITU-R BT.41931368-8, 1992.

[29] C. Li, S. Telemi, X. Zhang, R. Brugger, I. Angulo, and P. Angueira, "Planning Large Single Frequency Networks for DVB-T2," IEEE Trans. Broadcast., to appear.

[30] 3GPP TR 25.814 V7.1.0, Physical layer aspect for evolved Universal Terrestrial Radio Access (UTRA).

[31] S. Szyszkowicz, H. Yanikomeroglu, and J. Thompson, "On the Feasibility of Wireless Shadowing Correlation Models," IEEE Trans. Veh. Technol., vol. 59, no. 9, pp. 4222-4236, Nov. 2010.

[32] A. Ligeti, "Outage Probability in the Presence of Correlated Lognormal Useful and Interfering Components," IEEE Commun. Lett., vol. 4, no. 1, pp. 15-17, Jan. 2000.

[33] C. L. J. Lam and T. Le-Ngoc, "Log-Shifted Gamma Approximation to Lognormal Sum Distributions." IEEE Trans. Veh. Technol., vol. 56, no. 4, pp. 2121 - 2129, Jul. 2007.

[34] S. C. Schwartz and Y. S. Yeh, "On the Distribution Function and Moments of Power Sums with Log-normal Components," Bell Syst. Tech. J., vol. 61, no. 7, pp. 1441-1462, Sep. 1982.

[35] A. Safak, "Statistical analysis of the power sum of multiple correlated Log-normal Components," IEEE Trans. Veh. Technol., vol. 42, no. 1, pp. 58-61, Feb. 1993.

[36] V. Petrini and H. R. Karimi, "Tv white space databases: Algorithms for the calculation of maximum permitted radiated power levels," in Proceedings of IEEE DySPAN, 2012.

[37] RRC-06, "Final Acts for planning of the digital terrestrial broadcasting service in parts of Regions 1 and 3, in the frequency bands 174-230 MHz and 470-862 MHz," 2006.

[38] FCC LEARN Program - Statistics of Full Power and Class A Television Stations in the United States as of February 22, 2012 - Report No. TAB 2013-01, Jul. 16, 2013.

[39] U. Meabe, X. Gil, C. Li, M. Velez, and P. Angueira, "On the Coverage and Cost of HPHT vs LPLT Networks for Rooftop, Portable, and Mobile Broadcast Services Delivery," IEEE Trans. Broadcast., to appear.

[40] ITU-R, "Method for point-to-area predictions for terrestrial services in the frequency range $30 \mathrm{MHz}$ to $3000 \mathrm{MHz}$ ", Recommendation ITU-R P.1546-5, Sep. 2013.

[41] U.S. Energy Information Administration, "Average Retail Price of Electricity to Ultimate Customers by End-Use Sector [Online]," in http://www.eia.gov/electricity/.

[42] White House: Office of Management and Budget, "Circular A-94 Appendix C: Discount rates for cost-effectiveness, lease purchase, and related analyses [Online]," in http://www.whitehouse.gov/omb.

[43] FCC Staff Technical Paper, "Mobile broadband; the benefits of additional spectrum," October 2010.

[44] FCC Auction Summaries, Wireless Telecommunications Bureau, in FCC website, wireless.fcc.gov, 2015.

[45] United States Census Bureau, in www.census.gov, 2010. 
[46] CEA Press Release, "Smartphones, HDTVs Are the Most Planned CE Purchases This Year, According to CEA Study [Online]," in $\quad h t t p s: / / w w w . c e . o r g / N e w s / N e w s-R e l e a s e s / P r e s s-R e l e a s e s / 2012-$ Press-Releases/Smartphones,-HDTVs-Are-the-Most-Planned-CEPurchas.aspx, Apr. 2012.

[47] TVTechnology, "CEA says 7 percent watch OTA TV only; NAB cries foul [Online]," in http://www.tvtechnology.com/news/0086/ceasays-percent-watch-ota-tv-only-nab-cries-foul/225502, Aug. 2013.

[48] TVNewsCheck, "Path to Next-Gen Broadcasting Not Easy [Online]," in http://www.tvnewscheck.com/article/82140/path-to-nextgenbroadcasting-not-easy, Jan. 2015. 